\title{
NOTE TAXONOMIQUE \\ SUR EPEORUS TORRENTIUM EATON, 1881 \\ ET E. ASSIMILIS EATON, 1885 \\ [Ephemeroptera, Heptageniidae]
}

par C. Berthélemy et A. Thomas.

\section{1. - INTRODUCTION}

Eaton décrivit Epeorus torrentium en 1881 et $E$. assimilis en 1885. La première espèce avait été capturée dans les Pyrénées et Eaton y rapporta en outre, avec réserve, un subimago femelle provenant du nond de l'Italie. Pour $E$. assimilis, il cita, comme régions d'origine, les Pyrénées, la Belgique et la Forêt-Noire. D'après les descriptions d'EAToN, les deux espèces différaient principalement par la taille et la coloration des sternites abdominaux.

En 1960, Kimmins désigna des lectotypes d'E. torrentium et d'E. assimilis et figura les genitalia de paratypes. Il indiqua que les différences entre ces genitalia n'étaient pas très évidentes et qu'il serait peut-être nécessaire de considérer ultérieurement $E$. torrentium et $\boldsymbol{E}$. assimilis comme des synonymes ou des sous-espèces.

Dans l'intervalle, $E$. assimilis figura dans les différentes faunes publiées en Europe centrale et orientale [KLAPALEK 1909, ULMER 1929, Schoenemund 1930, Mrkulski 1936, Bogoescu 1958, IAANda 1959, UjhELY 1 1959]. E. assimilis fut, en outre, fréquemment cité dans les listes faunistiques, ainsi que dans les travaux d'hydrobiologie des eaux courantes effectués dans ces mêmes régions.

Grandi [1960 a], dans sa faune d'Italie, reprit pour E. torrentium l'indication douteuse d'EATon citée ci-dessus. Elle nota aussi la présence de larves de cette espèce en Italie, larves sans doute déterminées d'après la description d'EATon [1885]. Comme cette description s'applique également à $E$. assimilis, l'existence d'E. torrentium en Italie devra être confirmée par des captures d'imagos.

En France, Lestage [1923], dans son catalogue, indiqua seulement les localités d'EATON. E. torrentium fut cité du Massif central par Verrier [1944, 1948 a, 1948 b, 1949, 1951], de l'Ariège, de l'Andorre et des Pyrénées-Orientales par Bertrand et Verrier [1950 a, b], et de ce dernier département par NicolaU-Guillaumet 
(1959, détermination Ch. Degrange). E. assimilis fut signalé de l'Ariège par Bertrand et Verrien [1950 b] et du Massif central par Fiasson [1964].

Bertrand et Verrier [1949], enfin, indiquèrent la présence d'E. alpicola Eaton, 1871 dans les Basses-Pyrénées et les Pyrénées centrales. Aucune des très nombreuses larves d'Epeorus capturées par nous dans la même région n'appartient à cette espèce. Il semble s'agir d'une confusion avec $\boldsymbol{E}$. torrentium, dont la coloration abdominale rappelle un peu celle qui est figurée pour $E$. alpicola dans les ouvrages de détermination cités ci-dessus.

La solution du problème posé par Kimmins [1960] devait donc être cherchée dans les Pyrénées et le Massif central :

- ou bien $E$. assimilis y était remplacé par $E$. torrentium avec, éventuellement, des formes intermédiaires,

- ou bien les deux formes coexistaient sans se mélanger.

L'examen de notre matériel nous a montré que le second terme de cette alternative était exact. Les Epeorus récoltés ont pu être classés en deux catégories distinctes et nos observations confirment ainsi la validité des deux espèces décrites par EAToN.

\section{2. - COMPARAISON DES DEUX ESPECES}

\section{1. - Genitalia (fig. 1 à 8).}

2.1.1. - D'avant en arrière, le pénis des Epeorus comprend deux expansions ventrales, une partie moyenne subcylindrique, à section ovale, et deux lobes latéraux.

La face ventrale des lobes latéraux est presque entièrement sclérifiée, à l'exception de la ligne médioventrale, de deux minces zones arquées situées dans l'axe de chaque lobe et de deux petites encoches distales (fig. 1 et 3, En.).

Les parties sclérifiées sont peu étendues sur la face dorsale : il existe deux sclérites parasagittaux (fig. 5 et 6,5 . ps.) et des sclérifications mal délimitées, situées de part et d'autre des encoches distales précitées. Les canaux éjaculateurs s'ouvrent sur la face dorsale (fig. 1 et 3 , Or.). On observe parfois une gouttière entre les orifices et les encoches distales non sclérifiées. Ce fait explique que les canaux éjaculateurs aient été prolongés jusqu'au bord distal sur les figures de Bogoescu [1958] et Grandr [1960 b].

La lame sclérifiée ventrale n'est pas plane, mais légèrement bombée. Par suite de la réduction des sclérifications dorsales, les deux lobes latéraux tendent à s'enrouler sur eux-mêmes et à se reployer vers le dos lors de la désiccation. Aussi l'aspect du pénis est-il assez variable au sein de chaque espèce. La figure d'UlmER 
[1929], par exemple, représente un individu plus desséché que celui qu'a dessiné Schoenemund [1930]. Nous donnons ici des figures d'organes éclaircis et aplatis, en préparation microscopique (fig. 1 et 3 ), et d'organes d'animaux conservés en alcool glycériné (fig. 5 à 8 ).
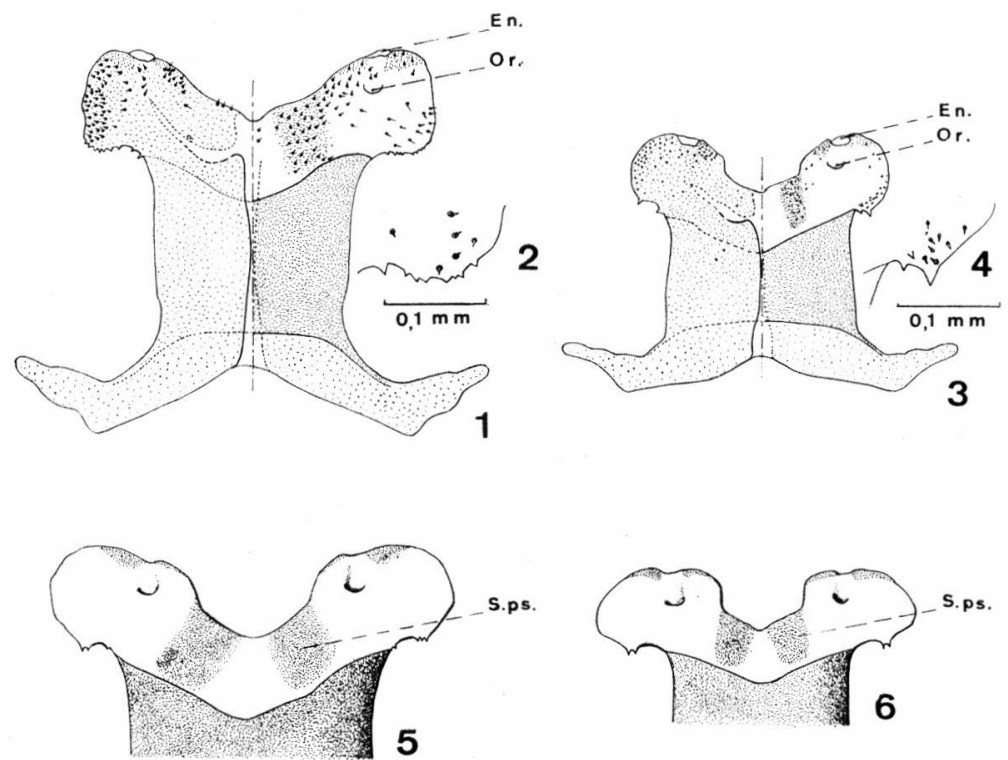

$1 \mathrm{~m} \mathrm{~m}$

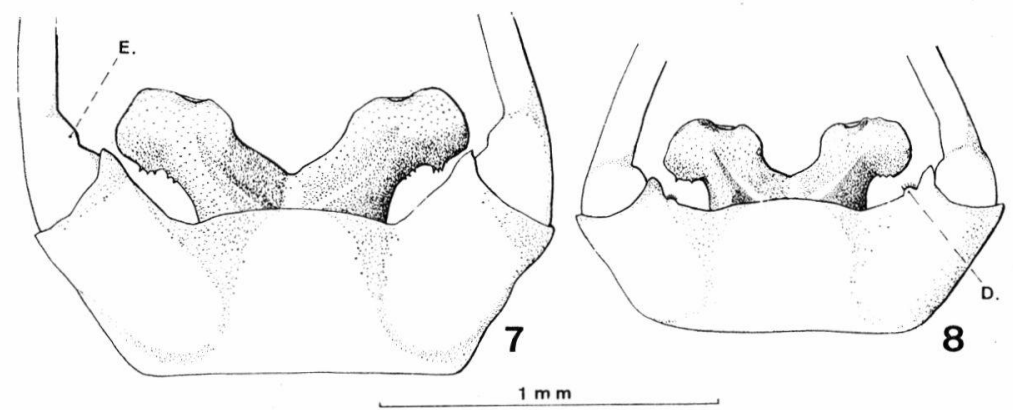

Fıg. 1 à 8. - Genitalia mâles d'Epeorus assimilis, à gauche (fig. 1, 2, 5 et 7), et d'E. torrentium, à droite (fig. $3,4,6$ et 8 ). FIG. 1 et 3 : pénis en préparation microscopique, après éclaircissement; à gauche de la ligne sagittale en tirets, vue ventrale; à droite de la ligne sagittale, vue dorsale; En. : encoche distale du lobe latéral droit. Or. : orifice du canal éjaculateur droit. Fig. 2 et 4 : portions des lobes latéraux vues à plus fort grossissement. FIG. 5 et 6 : moitié apicale du pénis d'exemplaires conservés en alcool glycériné, vue dorsale; $S$. ps. : sclérite parasagittal droit. Fig. 7 et 8 : Styligère, partie basale des styles et moitié apicale du pénis d'exemplaires conservés en alcool glycériné, vue ventrale; D. : denticulation du styligère; E. : épaississement du second article des styles. 
Le meilleur critère de différenciation des deux espèces est fourni par le contour de l'échancrure médiane située entre les deux lobes latéraux. Chez $E$. assimilis, cette échancrure est largement évasée, régulièrement concave et ses bords restent divergents (fig. 1,5 et 7 ). Chez $E$. torrentium, l'échancrure est anguleuse et les bords internes des lobes latéraux sont subparallèles dans leur moitié distale (fig. 3,6 et 8 ).

Cette différence est soulignée, sur la face dorsale, par la présence des sclérites parasagittaux, dont la limite externe est presque dans le prolongement du bord interne chez $E$. torrentium (fig. 6), alors qu'elle forme un angle net avec ce bond chez $E$. assimilis (fig. 5).

Chaque lobe latéral présente ainsi un «talon » mieux marqué chez $E$. torrentium que chez $E$. assimilis. Ce caractère apparaît lorsque l'on compare la figure d' $E$. torrentium donnée par EAton [1885] à celle d'E. assimilis donnée par Schoenemund [1930], ou encore les figures des paratypes étudiés par Kimmins [1960].

A fort grossissement, on observe que le nombre des denticulations présentes le long du bord externe des lobes latéraux, près de Ieur base, est plus élevé chez $E$. assimilis ( 3 à 13 denticulations de chaque côté, fig. 2) que chez $E$. torrentium (1 à 4 denticulations, fig. 4). Ces denticulations doivent être distinguées des poils plus ou moins épineux qui les entourent et que l'on retrouve sur la plus grande partie du pénis.

Quelques individus possèdent une ou deux denticulations analogues sur la partie centrale du pénis. Il est probable qu'il s'agit là des «petits titillateurs » dont la présence est signalée par Grand $[1960 \mathrm{~b}]$. Il est douteux qu'ils soient homologues des « titillateurs » que l'on observe chez les autres genres d'Heptageniidae.

2.1.2. - Les caractères des styles et du styligère sont moins constants. Chez $E$. assimilis, la base du second article des styles, qui est presque complètement soudé au premier, présente un épaississement interne (fig. $7, \mathrm{E}$.). La plupart des $E$. torrentium en sont dépourvus, mais deux de nos exemplaires en possèdent néanmoins.

De même, la petite denticulation de la face interne des prolongements latéraux du styligère signalée par Kimmins [1960] ne s'observe bien que chez les trois-quart environ de nos $E$. torrentium. Elle manque chez les autres, parfois unilatéralement, ou n'est marquée que par une augmentation de la densité des poils. 


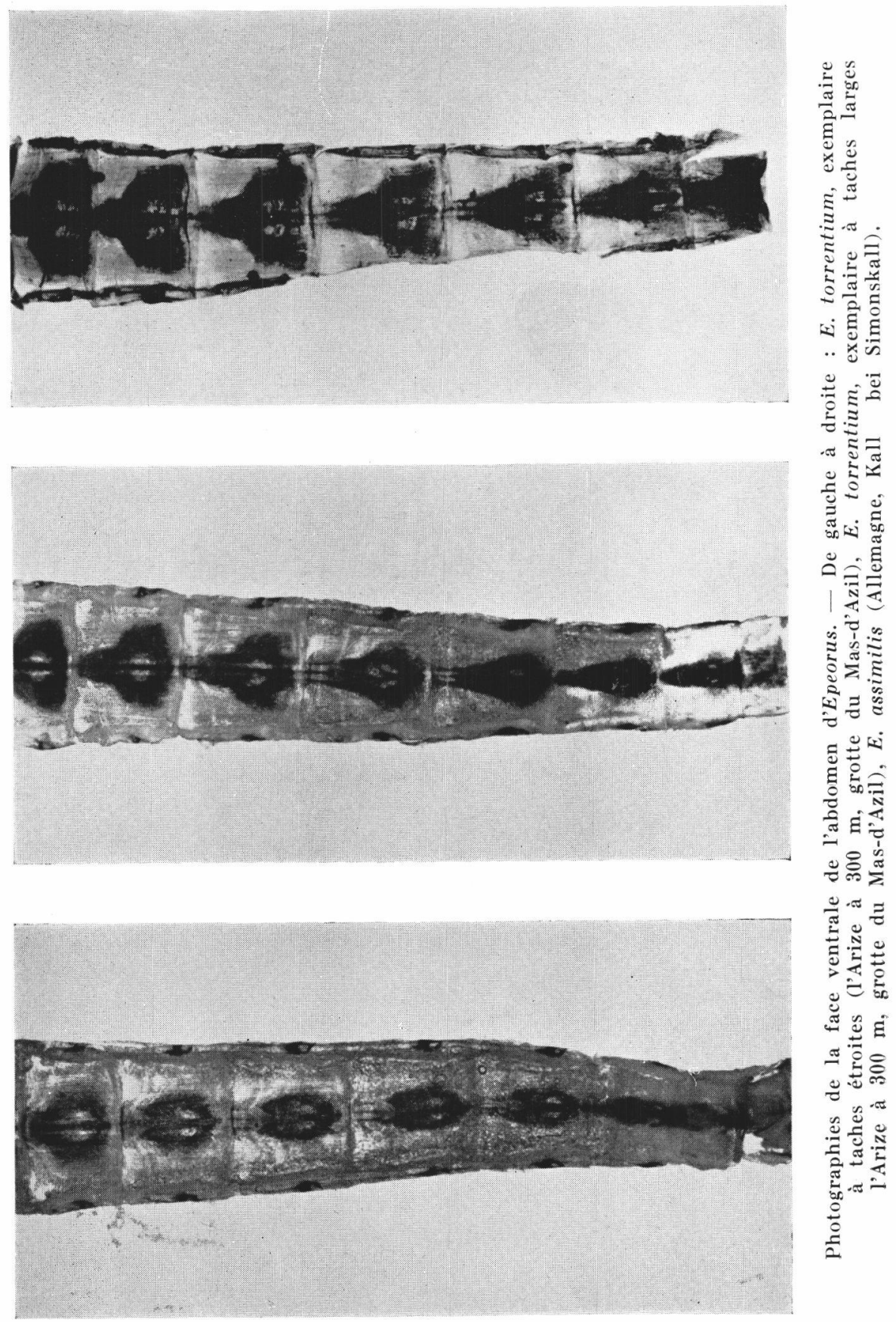



2.2. - Coloration ventrale (photographies hors-texte).

La tache ventrale brune est rétrécie dans le quart postérieur sur les sternites 3 à 7 d'E. torrentium, alors qu'elle y atteint sa largeur maximale chez $E$. assimilis. Ce critc̀re, proposé par EAton, s'applique bien à la majorité du matériel étudié. La largeur de la tache varie chez $E$. torrentium (phot. hors-texte), mais les angles postérieurs du sternite sont moins colorés que chez $E$. assimilis.

Chez $E$. assimilis, toutefois, la partie centrale, qui correspond à la seule partie pigmentée chez $E$. torrentium, est plus foncée que le reste de la tache ventrale. Chez beaucoup d'E. torrentium obtenus en élevage et chez quelques individus capturés dans la nature, la coloration ventrale est réduite à une petite tache arrondie médiane, située près du bord postérieur des sternites. En élevage, la mortalité des Epeorus est plus élevée que celle de la plupart des Ephéméroptères et il est probable que cette réduction de la pigmentation est due à des troubles du métabolisme. Comme un tel phénomène n'est pas exclu chez $E$. assimilis, la détermination des femelles à tache ventrale réduite est impossible lorsque le reste de la pigmentation est peu marqué.

\section{3. — Taille (fig. 9).}

La taille des $E$. torrentium est maximale au début de la période de vol. Ce fait a pu être mis en évidence au sein du matériel récolté près de l'Arize, à $300 \mathrm{~m}$ d'altitude, dans la Grotte du Mas-d'Azil (Ariège). Nous avons mesuré la longueur de l'aile antérieure, moins sujette que le corps à des déformations accidentelles.

Chez les imagos et subimagos mâles, l'aile mesure de $\mathbf{1 3 , 5}$ à $15,0 \mathrm{~mm}$ en mai ( 8 exemplaires, moyenne $14,40 \mathrm{~mm}$, erreur-type $0,201)$, de 11,5 à $14,5 \mathrm{~mm}$ en juin (37 exemplaires, moyenne $12,77 \pm 0,130 \mathrm{~mm}$ ), de 11,0 à $13,0 \mathrm{~mm}$ en juillet (23 exemplaires, moyenne $11,87 \pm 0,126 \mathrm{~mm}$ ) et de 11,0 à $13,2 \mathrm{~mm}$ en septembre et octobre ( 8 exemplaires, moyenne $12,18 \pm 0,306 \mathrm{~mm}$ ). Les différences entre les moyennes de mai, juin et juillet sont hautement significatives ( $p<0,01$, test $t$ de Student).

Chez les imagos et subimagos femelles, l'aile mesure de 16,2 à $16,5 \mathrm{~mm}$ en mai ( 3 exemplaires, moyenne $16,30 \pm 0,100 \mathrm{~mm}$ ), de 11,0 à $16,5 \mathrm{~mm}$ en juin (23 exemplaires, moyenne $14,53 \pm 0,245 \mathrm{~mm}$ ) et de 12,5 à $14,5 \mathrm{~mm}$ en juillet (15 exemplaires, moyenne 13,19 $\pm 0,167 \mathrm{~mm}$ ). Ici encore, les différences entre les moyennes de mai, juin et juillet sont hautement significatives. Il en est de même des différences entre les mâles et les femelles capturés pendant un même mois.

Lorsque l'altitude est plus élevée, la période de vol est plus tardive. Des larves provenant de cours d'eau de la Vallée d'Aure 
(Hautes-Pyrénées), situés entre 1500 et $2000 \mathrm{~m}$, ont été mises en élevages et quelques adultes ont été capturés dans la même région. En juillet, la taille des adultes de la Vallée d'Aure est la même que celle des exemplaires capturés en mai près de l'Arize à $300 \mathrm{~m}$; en août, elle correspond à celles des exemplaires capturés en juin près de l'Arize.

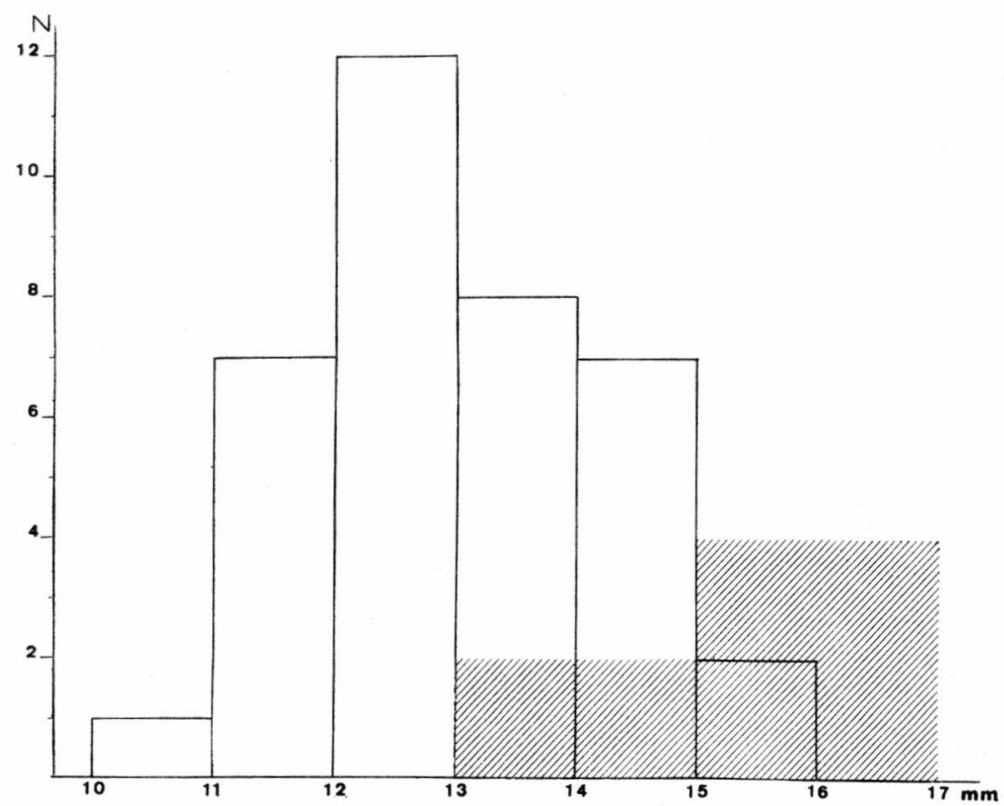

FIg. 9. - Longueur de l'aile antérieure chez Epeorus torrentium (histogramme en trait plein) et $E$. assimilis (histogramme hachuré); en abscisse : longueur de l'aile, en mm; en ordonnée : nombre d'individus.

Pour effectuer une comparaison détaillée de la taille d' $E$. torrentium et d'E. assimilis, il serait donc nécessaire de disposer de séries complètes de captures s'étendant sur l'ensemble des périodes de vol des deux espèces. Une telle série nous faisant défaut pour $E$. assimilis, nous avons construit un histogramme en utilisant tous les imagos mâles des deux espèces en bon état dont nous disposions (fig. 9). Les moyennes et erreurs-types sont de 12,75 $\pm 0,201 \mathrm{~mm}$ pour $E$. torrentium (37 exemplaires) et de 15,06 $\pm 0,272 \mathrm{~mm}$ pour $E$. assimilis (12 exemplaires). Si l'on admet que tous les $E$. assimilis n'ont pas été capturés au début de la période de vol, la différence est très nette $(\mathrm{P}<0,001)$. 


\section{4. - Larves.}

Les larves d'E. assimilis différeraient de celles d'E. torrentium par la forme de l'hypopharynx, selon š́mal [1924], et par la présence de denticulations à la face inférieure des griffes, selon Gauthier [1930]. Aucune de ces différences n'a été confirmée par l'examen de notre matériel. Il semble que les auteurs précités aient comparé des larves d'E. assimilis à des descriptions et des figures d'E. torrentium, et non des larves des deux espèces. Une comparaison détaillée des exuvies larvaires ne nous a point permis de mettre en évidence de critères spécifiques qui permettent la détermination des larves.

\section{3. - MATERIEL ETUDIÉ}

Outre notre propre matériel, nous avons pu étudier des Epeorus capturés en Allemagne et prêtés par $\mathbf{M}^{\mathrm{me}}$ Müllen-Liebenau, des exemplaires pyrénéens de la Collection Despax (Muséum d'Histoire Naturelle de Paris), communiqués par M Mie Kelner-Pillaut, des adultes et leurs exuvies obtenus en élevage par M. Thibault (Station d'Hydrobiologie Continentale de Biarritz), ainsi qu'une partie du matériel récolté dans la Massane (Pyrénées-Orientales) par M. Nicolau-Guillaumet. Nous prions ces correspondants d'accepter nos plus vifs remerciements pour l'aide qu'ils nous ont ainsi apportée.

\section{1. - Epeorus torrentium.}

\subsection{1. - Massif central.}

- Lozère : Monts d'Aubrac, cascade de Dérols, vers $1000 \mathrm{~m}, 21-\mathrm{V}-1964$, 2 imagos (i.) of, 2 subimagos (s.) ô, 3 i. $\$, 4$ s. $ᄋ$ (H. DÉcamps leg.).

\subsection{2. - Pyrénées.}

- Basses-Pyrénées : ruisseau du Lissuraga, entre 75 et $175 \mathrm{~m}, 21-\mathrm{IL} /$ 8-V-1966, 2 i. $\hat{f}, 1$ i. o, 3 s. $q$; ruisseau du Laurhibar, vers $200 \mathrm{~m}, 23-\mathrm{IL} /$ 11-IV-1966, 3 i. $\hat{\delta}, 1$ s. $\hat{\delta}, 1$ s. $q$; la Nivelle, vers $30 \mathrm{~m}, 18-\mathrm{X}-1966,1$ i. $\hat{s}$ (M. Thibault leg.).

- Hautes-Pyrénées : bassin hydrographique de la Neste d'Aure : déversoirs des Laquettes, vers $2000 \mathrm{~m}, 21 / 31$-VII-1965, 1 i. $\hat{\delta}, 1 \mathrm{~s}$. $\hat{\delta}, 1$ i. $?$ (A. T. leg.); déversoir de la tourbière Despax, vers $2100 \mathrm{~m}, 29$-VII-1964, 2 i. $q$ (H. Décamps leg.) ; Neste de Couplan, vers 1840 m, 1/31-VII-1965, 2 i. $\delta, 3$ i. $q$; Neste de Couplan, vers $1400 \mathrm{~m}, 21 / 31$-VIIIL1965, 1 i. $\delta$; Neste de Couplan, vers $1200 \mathrm{~m}, 1$-VII-1965, 31-VIII-1965, 2 i. $\hat{f}, 2$ s. $\hat{f}$, 6 i. $\circ, 2$ s. $q$ (A. T. leg.); ruisseau d'Espiaube, vers $815 \mathrm{~m}, 13$-VIII-1962, 1 of (C. B. leg.).

- Haute-Garonne : la Garonne vers $500 \mathrm{~m}, 10-\mathrm{IX}-1926,1$ i. $\hat{\delta}, 3$ i. ? (R. Despax leg.). 
- Ariège: le Lez à $430 \mathrm{~m}, 4$-VII-1963, 1 s. ๆ, 18-VII-1963, 1 i. ₹, 30-VII-1963, 1 i. \&; le Ribérot à $800 \mathrm{~m}, 24$-VII-1962, 1 i. ô; l'Arize à $300 \mathrm{~m}$ (grotte du Mas-d'Azil), 6-V-1963, 1 s. $\delta, 13-I X-1963,1$ i. $\hat{\delta}, 1$ s. $\delta$, 1-V-1964, 2 i. $\hat{\delta}, 6$ s. $\hat{o}, 3$ s. $+, 10-\mathrm{VI}-1964,5$ i. $\hat{\delta}, 4$ s. $\hat{\delta}, 2$ i.,+ 1 s. 20-V.HI-1965, 8 i. $\hat{\delta}, 16$ s. $\hat{\jmath}, 10$ i. $ᄋ, 5$ s. $ᄋ, 21-\mathrm{X}-1965,2$ i. $\hat{\delta}, 1$ s. $\hat{o}$ (C. B. leg.), 19-VI-1966, 4 i. $\delta, 24$ s. $\delta, 8$ i. $\uparrow, 14$ s. $ᄋ$ (J. Ferrieu leg.).

- Pyrénées-Orientales : le Cady, vers $700 \mathrm{~m}$, VII-1962, 1 i. $\&$ (N. BERTHÉlemy leg.); la Massane, vers $600 \mathrm{~m}, 5 / 28-\mathrm{V}-1958,2$ i. $\delta, 2$ i. $q$ (P. Nicolau-Guillaumet leg.).

\section{2. - Epeorus assimilis.}

3.2.1. - Allemagne.

Kall bei Simonskall, 8-VI-1964, 5 i. $\hat{\jmath}, 1$ i. $q$ (I. Müller-Liebenau leg.).

3.2.2. - Massif central.

- Cantal : vallée de la Rhue-de-Cheylade, vers $1000 \mathrm{~m}, 23-\mathrm{V}-1964,1$ (H. Décamps leg.).

3.2.3. - Pyrénées.

-- Hautes-Pyrénées: Neste d'Aure à $1700 \mathrm{~m}, 17-\mathrm{VII}-1925,1$ i. \& (R. Despax leg.) ; Neste d'Aure à $1200 \mathrm{~m}, 1 / 7-V H$-1965, 1 i. ô (A. T. leg.). leg.).

- Haute-Garonne : la Pique vers $1000 \mathrm{~m}, 29-\mathrm{VI}-1925,3$ i. $\hat{o}$ (R. Drspax

— Ariège : l'Hers Vif à $605 \mathrm{~m}, 5$-VII-1963, 1 i. ô (C. B. leg.).

\section{4. - CONCLUSION}

La coexistence dans le Massif central et les Pyrénées de deux formes distinctes par les genitalia, la coloration abdominale et la taille confirme la validité des espèces décrites par Eaton.

Les points suivants, toutefois, devront être précisés :

- au point de vue taxonomique, les relations d'E. assimilis et d'E. torrentium avec E. sylvicola (ED. Pictet), du Portugal;

- au point de vue biogéographique, l'extension éventuelle de l'aire de répartition des deux espèces dans la Péninsule Ibérique, ainsi que la limite orientale exacte de l'aire d' $E$. torrentium, qui coïncide peut-être avec les vallées de la Saône et du Rhône;

- au point de vue écologique, les caractères propres aux habitats de ces deux espèces. Aucune des principales différenciations mises en évidence chez les espèces voisines de Plécoptères et de Coléoptères des Pyrénées [Berthélemy 1966] ne semble applicable ici. $\mathrm{Ni}$ l'altitude, ni le débit, ni la pente, ni la nature des terrains, ni, enfin, les périodes de vol ne permettent d'opposer nettement les deux espèces. 


\section{RÉSUME}

Epeorus torrentium et $E$. assimilis sont deux bonnes espèces, étroitement apparentées, qui coexistent dans le Massif central et les Pyrénées. Elles diffèrent par la forme de l'échancrure distale entre les lobes latéraux du pénis (fig. 1 à 8 ), plus largement évasée et moins anguleuse chez E. assimilis, par la coloration des sternites abdominaux (phot. hors-texte) et par la taille (fig. 9).

\section{TAXONOMU NOTE ON EPEORUS TORRENTIUM EATON, 1881 AND E. ASSIMILIS EATON, 1885}

[Ephemeroptera, Heptageniidae]

Epeorus lorrentium and $E$. assimilis are two valid species, closely related, which coexist in the French « Massif central» and in the Pyrenees. They differ by the shape of the distal notch between the lateral lobes of the penis, broader and less angular in E. assimilis (fig. 1 to 8), by the pigmentation of the abdominal sterna (phot.) and by their size.

\section{TAXIONOMISCHE UNTERSUCHUNG VON EPEORUS TORRENTIUM EAtoN, 1881 UND E. ASSIMILIS EATON, 1885 \\ [Ephemeroptera, Heptageniidae]}

Epeorus torrentium und $E$. assimilis sind zwei gültige Arten, nah verwandt mit einander, die im französischen «Massif Central 》 und in den Pyrenäen zusammenleben. Sie unterscheiden sich durch die Form der distalen Ausbuchtung zwischen den beiden Penisloben, weiter und weniger kantig bei $E$. assimilis $(A b b, 1$ bis 8$)$, durch die Färbung der Unterseite des Hinterleibes (Phot.) und durch ihre Größe.

\section{TRAVAUX CITES}

BERTHÉlemy (C.). 1966. - Recherches écologiques et biogéographiques sur les Plécoptères et Coléoptères d'eau courante (Hydraena et Elminthidae) des Pyrénées. Annls Limnol., 2 : 227-458.

Bertrand (H.) et Verrier (M.-L.). 1949. - Contribution à la Biogéographie des Ephéméroptères des Pyrénées. Bull. biol. Fr. Belg., 83 : 1-24.

Bertrand (H.) et Verrier (M.-L.), 1950 a. - Contribution à l'étude de la faune des eaux douces de la région orientale des Pyrénées : Ephéméroptères. Vie Milieu, 1 : 217-234.

Bertrand (H.) et Verrier (M.-L.). 1950 b. - - Contribution à l'étude de la faune des eaux douces de la région orientale des Pyrénées : Ephéméroptères (Deuxième partie). Vie Milieu, 1 : 449-459.

Bogoescu (C.). 1958. - Ephemeroptera. In Fauna Republicii Populare Romine, Y (3) : 189 p. Bucuresti.

Eaton (A. E.).1881-1882. - An announcement of new genera in the Ephemeridae. Entomologist's mon. Mag. 17 : 191-197, $18: 21-27,207-208$. 
Eaton (A. E.). 1883-1888. - A revisional monograph of recent Ephemeridae or Mayflies. Trans. Linn. Soc. Lond., Zoology, $3: 1352+$ $65 \mathrm{pl}$.

Fiasson (S.). 1964. - Recherches écologiques sur la faune d'une rivière de la région lyonnaise : l'Yzeron (Thèse sc. Lyon), $182 \mathrm{p}$.

Gauthier (M.). 1930. - Etude descriptive de la nymphe et de l'adulte d' «Epeorus alpicola》Eat. Trav. Lab. Hydrobiol. Piscic. Univ. Grenoble, $22: 185-196+5 \mathrm{pl}$.

Grandi (M.). 1960 a. - Ephemeroidea. In Fauna d'Italia, 3 : 472 p. Bologna.

Grandi (M.). 1960 b. - Contributi allo studio degli Efemeroidei italiani. XXIII. Gli organi genitali esterni maschili degli Efemeroidei. Boll. Ist. Ent. Univ. Bologna, 24 : 67-120.

Kimmins (D. E.). 1960. - The Ephemeroptera types of species described by A. E. Eaton, R. Mc IAACHian and F. Walker, with particular reference to those in the British Museum (Natural History). Bull. Br. Mus. nat. Hist., Entomology, 9 (4) : 269-318.

Klapalek (F.). 1909. - Ephemerida, Eintagsfliegen. In Die Süsswasserfauna Deutschlands, 8 (1) : 32 p. Jena.

I.ANDA (V.). 1959. - Ephemeroptera. In Klíč $k$ faunĕ ¿SR. $3: 143-167$, Praha.

Lestage (J.-A.). 1923. - Catalogue des Ephémères de France. Annls Soc. ent. Fr., 91 [1922] : 273-276.

Mikulski (J. S.). 1936. - Jetki (Ephemeroptera). In Fauna Stodkowodna Polski, 15 : 168 p. Warszawa.

Nicolau-Guillaumet (P.). 1959. -- Recherches faunistiques et écologiques sur la rivière «La Massane». Vie Milieu, $10: 217-266$.

Šamal (J.). 1924. - Nymphy ceskych jepic. IV. Epeorus assimilis EtN. Čas. čsl. Spol. Ent., 21 : 42-45.

Schoenemund (E.). 1930. - Eintagsfliegen oder Ephemeroptera. In Die Tierwelt Deutschlands, 19 : 106 p. Jena.

Uлhely (S.). 1959. - Ephemeroptera -Kérészek. In Fauna Hungariae, 49 : 96 p. Budapest.

Uimer (G.). 1929. - Ephemeroptera. In Die Tierwelt Mitteleuropas, $4: 43$ p. Leipzig.

Verrier (M.-I.). 1944. - Nouvelles stations françaises d'Ephéméroptères. Bull. Soc. ent. Fr., [1944] : 27-30.

VeRhIER (M.-L.) 1948 a. - L'oxygène dissous et la répartition des larves d'Ephémères. C. R. hebd. Séanc. Acad. Sc., Paris, 226 : 1548-1550.

Verrier (M.-L.). 1948 b. — Nouvelles stations françaises d'Ephémères (Deuxième note). Bull. Soc. ent. Fr., [1948] : 66-70.

Verrier (M.-L.). 1949. - Les facteurs de la répartition des Ephémères. C. $R$. somm. Séanc. Soc. Biogéogr., 26 (226) : 38-41.

Verrier (M.-L.). 1951. - Ecologie et répartition des Ephéméroptères du Massif Central. C. R. hebd. Séanc. Acad. Sc., Paris, 232 : 1700-1702.

(Laboratoire de Zoologie de la Faculté des Sciences, 118, route de Narbonne, 31-Toulouse.) 